\title{
Revisão integrativa: sofrimento psíquico em estudantes universitários e fatores associados
}

\author{
Integrative review: psychological distress among university students \\ and correlated factors
}

Karen Mendes Graner (https://orcid.org/0000-0002-7176-5778) ${ }^{1}$

Ana Teresa de Abreu Ramos Cerqueira (https://orcid.org/0000-0003-2374-8890) ${ }^{1}$

${ }^{1}$ Departamento de Neurologia e Psiquiatria, Faculdade de Medicina de Botucatu, Universidade Estadual Paulista Júlio de Mesquita Filho. Av. Prof. Mário Rubens Guimarães Montenegro $s / \mathrm{n}$,

Distrito de Rubião Júnior. 18618-970 Botucatu

SP Brasil.

kmendesgra@gmail.com

\begin{abstract}
This review sought to identify risk factors and protection from psychic distress among university students. Empirical studies were analyzed in the Web of Science, Medline and Scopus databases. A total of 1,375 articles were located, and after the exclusion criteria were applied, 37 articles made up the final sample, most of which were cross-sectional studies published in the last five years, in developed countries and with students in the health area. The most frequently used instruments for psychic distress screening were the General Health Questionnaire and the Self Reporting Questionnaire, which identified a prevalence of between $18.5 \%$ and $49.1 \%$ and, as risk factors, conditions related to academic life (24) and to health (22). Twelve studies identified as protective factors: given coping strategies, sense of coherence, self-efficacy, vigor, self-esteem, resilience, among other psychological conditions. This review singles out directions for possible interventions that could contribute to the well-being of students and to stimulate more positive experiences in the educational environment.
\end{abstract}

Key words Psychic distress, Mental disorders, Students, Risk factors, Review
Resumo Esta revisão propôs-se a identificar fatores de risco e proteção para sofrimento psíquico em estudantes universitários. Analisaram-se estudos empiricos nas bases de dados: Web of Science, Medline e Scopus. Foram localizados 1375 artigos e, aplicados os critérios de exclusão, 37 artigos compuseram a amostra final, tendo sido a maioria estudos transversais, publicados nos últimos cinco anos, em países desenvolvidos, com estudantes da área da saúde. Os instrumentos mais utilizados para o rastreamento de sofrimento psíquico foram $o$ General Health Questionnaire $e o$ Self Reporting Questionnarie, que identificaram prevalências de $18,5 \%$ a 49,1\% e, como fatores de risco, condições relativas à vida acadêmica (24) e à saúde (22). Foram identificadas como fatores de proteção, em doze estudos, apresentar determinadas estratégias de coping, senso de coerência, autoeficácia, vigor, autoestima, resiliência, entre outras condições psicológicas. Essa revisão aponta direções para possivieis intervenções que poderiam contribuir para o bem estar dos estudantes e para estimular vivências mais positivas no ambiente educacional.

Palavras-chave Sofrimento psíquico, Transtornos mentais, Estudantes, Fatores de risco, Revisão 


\section{Introdução}

Sofrimento psíquico entre estudantes universitários tem sido foco da literatura em saúde. Aproximadamente, $30 \%$ dos adultos brasileiros apresentaram transtornos mentais comuns (TMC) $)^{1}$, como encontrado em pesquisa com adolescentes ${ }^{2}$. Porém, a prevalência deste sofrimento varia segundo a população estudada e os métodos utilizados nas pesquisas ${ }^{3}$. Estudos realizados com universitários brasileiros, especialmente os da área da saúde, indicam variação de TMC de $18,5 \%$ a $44,9 \%^{4-11}$. TMC são estados mistos de depressão e ansiedade, caracterizados pela presença de sintomas como insônia, fadiga, irritabilidade, esquecimento, dificuldade de concentração e queixas somáticas ${ }^{12} \mathrm{e}$ podem ser investigados por instrumentos de screening. Apesar desses sintomas não atingirem as exigências para serem considerados como transtornos psiquiátricos (DSM-V ${ }^{13}$; CID-10 ${ }^{14}$ ) afetam negativamente a vida das pessoas ${ }^{12}$.

O universitário vivencia mudanças biológicas, psicológicas e sociais ${ }^{15}$ e se depara com aspectos estressores durante a vida acadêmica. Nos cursos de saúde, o início da prática clínica e a proximidade com o sofrimento e a morte são potenciais estressores ${ }^{15-18}$. Destaca-se que o sofrimento psíquico entre estudantes pode associar-se à percepção negativa do ambiente acadêmico e à queda na qualidade de vida ${ }^{19,20}$.

A investigação das características associadas à TMC em alunos possibilita que fatores de risco e proteção sejam identificados, propiciando ações preventivas e de promoção da saúde ${ }^{21-24}$. Estudos mostram associação de sofrimento com características, como sexo feminino, ser mais jovem, ter baixa renda, baixo apoio social, dificuldade para fazer amigos, avaliar seu desempenho acadêmico como ruim e pensar em abandonar o curso ${ }^{16,25-27}$. A percepção dos estudantes sobre o ambiente educacional e sua associação com sofrimento psíquico também vem sendo investigado através de instrumentos padronizados ${ }^{28-33}$. Além disso, a inclusão de aspectos psicológicos, como coping e resiliência, mais recentemente, visam identificar fatores de proteção ${ }^{32,34,35}$. No entanto, os resultados dessas pesquisas ainda são inconclusivas.

Este estudo propõe-se a identificar, por meio de revisão integrativa, os fatores de risco e de proteção para sofrimento psíquico entre estudantes universitários em produções disponíveis na literatura científica nacional e internacional.

\section{Método}

Esta revisão integrativa ${ }^{36}$ foi desenvolvida a partir de proposta de Ganong ${ }^{37}$, que estabelece cinco passos: (1) seleção do tema/pergunta; (2) estabelecimento de critérios de inclusão; (3) definição das informações a serem extraídas dos estudos; (4) avaliação dos estudos; (5) interpretação dos resultados; (6) apresentação da revisão $0^{36,37}$. Para atender esses passos, identificou-se o tema e elaborou-se a pergunta central da presente pesquisa: Quais fatores (de risco e proteção) encontram-se associados ao sofrimento psíquico entre universitários, segundo literatura nacional e internacional?

A busca dos dados foi realizada em novembro de 2016. Foram utilizadas três bases de dados: Web of Science, Medline e Scopus. Para garantir o controle de vocabulário e identificação de palavras correspondentes, utilizou-se o Medical Subject Heading Terms (Mesh Terms). Foram elaborados três grupos de palavras-chaves, combinadas pelo método boleano OR e AND: ("common mental disorder" or "psychological distress" or "psychological symptoms") and (students or "college students" or "undergraduate students" or "university students") and ("socioeconomic factors" or "socioeconomic factor" or "sociodemographic characteristics" or sociodemographic or "social support" or "psychosocial aspects" or "educational achievements" or "educational achievement" or "educational environment" or "coping behaviors" or "coping behavior" or "coping skills" or "coping skill" or coping or "psychological resilience" or resilience or "psychological factors" or "psychological factor" or "psychological determinants" or "psychological aspects").

A partir desse conjunto de palavras-chave e para a busca dos artigos que integrariam esta revisão, através dos filtros das próprias bases de dados, estabeleceu-se os seguintes critérios de inclusão: ano de publicação (2006-2016) e língua (português, inglês ou espanhol). Cada referência foi importada para o Software EndNote, através do qual foram excluídas as repetições entre as bases de dados. Após isto, as referências foram transferidas para o Programa Excel-Windows 10, verificando novamente as duplicações.

A primeira etapa da avaliação das referências incluídas constou da leitura dos títulos dos artigos, a segunda da leitura dos resumos, e a terceira e última etapa da leitura e avaliação na íntegra dos estudos. Em todas as etapas, a leitura e a análise dos artigos foram realizadas por dois pesquisadores 
independentes para evitar vieses de seleção, obtendo-se $90 \%$ de concordância entre revisores. Eventuais discordâncias foram discutidas e avaliadas conjuntamente para se estabelecer um consenso.

$\mathrm{Na}$ primeira e na segunda etapas excluíramse os estudos cuja população alvo não era de universitários, em que o desfecho não era sintomas psicológicos, não eram estudos empíricos e não estavam disponíveis para acesso. Na terceira etapa, os artigos foram lidos na íntegra e, para se verificar a pertinência de sua inclusão, utilizou-se as informações: curso acadêmico, total da amostra, país da pesquisa, instrumentos utilizados para medida do desfecho, prevalência de sofrimento psíquico e associações estatisticamente significativas entre as variáveis independentes e desfecho. A partir disso, observou-se uma variabilidade de desfechos investigados (TMC, sintomas ansiosos, depressivos, estresse, e outros transtornos mentais) e de instrumentos utilizados. Optouse pela inclusão dos estudos que apresentavam como desfecho sofrimento psíquico, em geral, ou $\mathrm{TMC}^{12}$ avaliados por instrumentos de screening padronizados. Considerou-se que esses critérios favoreceriam a obtenção da resposta à pergunta desta revisão. Ambos (restrição do desfecho e uso de instrumentos) foram estabelecidos considerando-se os resultados obtidos por Patel et al. ${ }^{38}$ que mostraram alta sensibilidade de alguns instrumentos de screening de TMC, em especial, o GHQ-12 e o SRQ-20.

\section{Resultados}

Identificaram-se 1.375 publicações, 568 no Web of Science, 180 no Medline, e 627 na base de dados Scopus. Após a aplicação do critério de ano de publicação e idioma, permaneceram 401 estudos na Web of Science, 122 na Medline e 390 na Scopus. Destes, foram encontradas 281 duplicações, permanecendo 632 estudos. Na primeira etapa, foram excluídos 453 estudos, permanecendo 179. Na segunda etapa, foram selecionados 89 estudos, dos quais 18 não estavam disponíveis para livre acesso nas bases de dados, dois foram excluídos por não apresentarem desfecho sofrimento e dois por não terem universitários como população alvo. Após leitura na íntegra dos artigos, permaneceram 67 estudos; porém, optou-se por excluir 29 que apresentavam como desfecho sintomas específicos e mais um por não ter sido utilizado instrumento padronizado para avaliação de sofrimento psíquico, compondo, assim, amostra final de 37 artigos (Figura 1).
Conforme Tabela 1, 59\% foram publicados nos últimos cinco anos, sendo 25\% em 2014. Predominaram estudos transversais $(97,3 \%)$, realizados em países desenvolvidos (59,5\%). Do total, $69,4 \%$ investigaram fatores associados à TMC e $35,1 \%$ investigaram sofrimento psíquico em geral. Os instrumentos mais utilizados foram: $G e$ neral Health Questionaire - GHQ-20, Self repporting Questionaire - SRQ-20 e Kessler Psychological Distress Scale - K-10. Predominaram pesquisas com estudantes da saúde (medicina, odontologia, enfermagem e outros). Em 14 artigos não foi possível obter essa informação.

No Quadro 1, as prevalências de TMC obtidas pelo GHQ-12 variaram de 18,5\% a 48,7\%, sendo inferiores a $20 \%$ em estudos realizados com alunos de medicina ${ }^{39,40}$, na Hungria. Identificou-se prevalências de 20 a $40 \%$ em cinco estudos, sendo três realizados em países desenvolvidos (enfermagem $)^{41-43}$ e dois em países em desenvolvimento (enfermagem e fisioterapia; e em outro não foi possível identificar a população $)^{44,45}$. Outros cinco estudos, realizados em países desenvolvidos, encontraram prevalências superiores a $40 \%{ }^{46-50}$. Dados obtidos pelo SRQ-20, indicaram variação nas prevalências de sofrimento psíquico de 33,7 a $49,1 \%$, sendo em seis pesquisas brasileiras ${ }^{7-11,51}$ de 33,7 a 44,9\% e em dois na Etiópia ${ }^{52,53}$ de 40,9 a $49,1 \%$. O instrumento K-10 foi utilizado em cinco pesquisas ${ }^{54-59}$, com prevalências acima de $50 \%$ de sofrimento em dois estudos com alunos de medicina na Arábia Saudita $(57 \% \text { e } 63 \%)^{54,55}$ e abaixo de $30 \%$ em estudo realizado nos EUA $^{56}$ e Austrália ${ }^{57}$.

Os fatores associados a sofrimento psíquico/ TMC entre estudantes foram agrupados em seis categorias: Sociodemográficas - idade, sexo, arranjo de moradia, dados familiares, renda e religiosidade; Saúde - condições e percepções sobre a sua saúde, estilo de vida e histórico de tratamentos psicológico/ou psiquiátrico; Relacionais - percepção sobre relacionamentos sociais/apoio social; Acadêmicas - características do curso, percepções sobre o curso, ambiente educacional e desempenho acadêmico; Psicológicas - traços de personalidade, estratégias de coping e resiliência; e Sociais/Violência - discriminação e violência social.

$\mathrm{Na}$ Tabela 2, observa-se que as variáveis mais frequentes como fatores de risco para sofrimento psíquico foram as classificadas na categoria Acadêmicas (24 estudos), chamando-se a atenção para a subcategoria série do curso (três referindo-se às primeiras e dois às últimas séries), ter pensado em abandonar o curso, excesso de ho- 


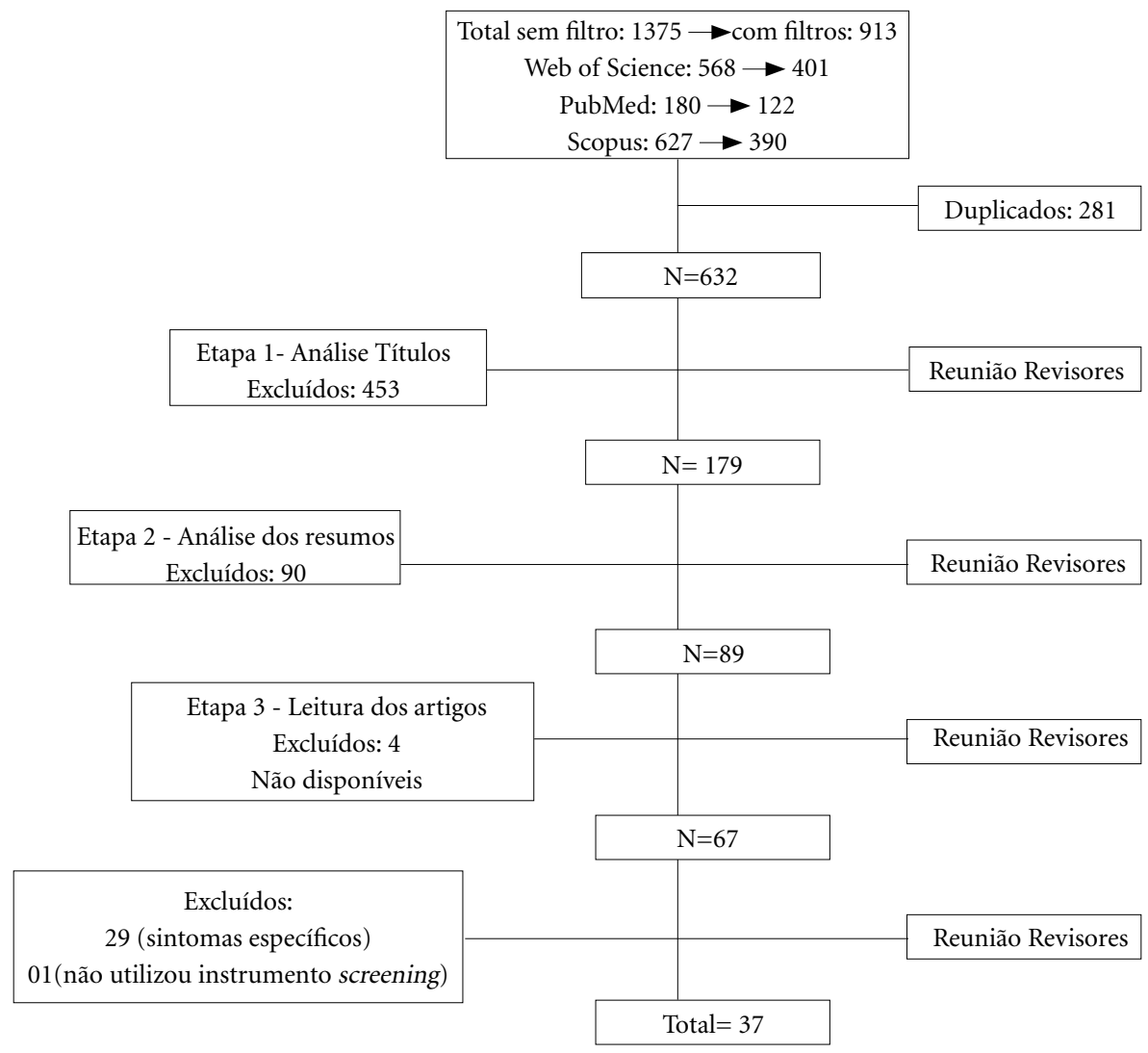

Figura 1. Fluxograma referente às etapas da seleção dos estudos pelos revisores.

ras de estudo ou dificuldade para conciliar estudo, lazer/descanso em dois estudos, assim como apontar o curso como fonte de estresse/tensão, ter expectativas ruins em relação ao futuro profissional, insatisfação com o curso/ter pouco interesse pelo mesmo e sentir desconforto durante as avaliações. Em relação aos fatores inseridos na categoria Saúde (22), destaca-se maior sofrimento entre os que afirmaram ser tabagista, ter problemas de saúde, não realizar atividade física e vivenciar estresse. Em relação aos fatores identificados na categoria Sociodemográficas (18), a terceira mais frequente como risco para sofrimento psíquico, destacam-se sexo feminino, ter maior idade e baixa renda. Além dessas, associaram-se significativamente as subcategorias: não ter religião, residir em área sem saneamento básico, ter filho em idade escolar, e não ter companheiro(a). Entre as características inseridas na categoria $R e$ lacionais (15), destaca-se: ter dificuldade no relacionamento com os amigos, não se sentir adaptado à vida acadêmica, baixo apoio social (medido/ referido), sentir-se rejeitado pelos amigos e não receber apoio emocional. Na categoria Psicológicas também foram identificadas como risco (9): estratégias de coping focalizadas na emoção, descarga emocional (sentimentos negativos), $c o-$ ping forbearance (não compartilhar problemas), coping negativo/destrutivo (comer muito/gastar dinheiro), neuroticismo, perfeccionismo e baixa autoestima. Por fim, na categoria Social/Violência, como ter sofrido discriminação (racial, idade, classe), perceber o clima da universidade como tenso em relação à discriminação (gênero, raça, orientação sexual), ter tido preocupação com a segurança pessoal e ter sofrido agressão estiveram associados a sofrimento psíquico.

$\mathrm{Na}$ Tabela 3, destacam-se as características identificadas como fatores de proteção para sofrimento dos alunos: Psicológicas (12), como: $\mathrm{co}-$ ping focalizado no problema, ter referido coping positivo (conversar com amigos, ter atividades de lazer), senso de coerência, autoeficácia, afeto positivo, autoestima, resiliência, extroversão, do- 
Tabela 1. Frequência absoluta e porcentagens das características dos estudos incluídos $(\mathrm{n}=37)$.

\begin{tabular}{|c|c|c|c|}
\hline Variáveis & Categorias & $\mathbf{n}$ & $\%$ \\
\hline \multirow[t]{11}{*}{ Ano } & 2006 & 2 & 5,4 \\
\hline & 2007 & 3 & 8,1 \\
\hline & 2008 & 4 & 10,8 \\
\hline & 2009 & 2 & 5,4 \\
\hline & 2010 & 4 & 10,8 \\
\hline & 2011 & 3 & 8,1 \\
\hline & 2012 & 3 & 8,1 \\
\hline & 2013 & - & - \\
\hline & 2014 & 10 & 27,0 \\
\hline & 2015 & 3 & 8,1 \\
\hline & 2016 & 3 & 8,1 \\
\hline \multirow[t]{2}{*}{ Delineamento } & Transversal & 36 & 97,3 \\
\hline & Coorte & 1 & 2,7 \\
\hline Países IDH Muito Alto & Desenvolvidos & 22 & 59,5 \\
\hline Países IDH Alto e Médio & Em desenvolvimento & 13 & 35,1 \\
\hline Países IDH muito Muito Baixo & Subdesenvolvidos & 2 & 5,4 \\
\hline \multirow[t]{2}{*}{ Desfecho } & Sofrimento psíquico & 13 & 35,1 \\
\hline & TMC & 24 & 64,9 \\
\hline \multirow[t]{9}{*}{ Instrumentos } & General Health Questionaire-12 (GHQ-12) & 16 & 43,2 \\
\hline & Self Reporting Questinaire-20 (SRQ-20) & 8 & 21,6 \\
\hline & Kessler Psychological Distress Scale-10 (K-10) & 5 & 13,5 \\
\hline & Mental Health -5 (MH-5) & 2 & 5,4 \\
\hline & Brief Symptom Checklist-53 (SC-53) & 2 & 5,4 \\
\hline & Overall Mental Health Index (MHI) & 1 & 2,7 \\
\hline & Symptom Checklist-90 (SC-90) & 1 & 2,7 \\
\hline & Outcome Questionnaire-45 (OQ-45) & 1 & 2,7 \\
\hline & Hopkins Symptom Checklist (HSCL) & 1 & 2,7 \\
\hline \multirow[t]{8}{*}{ Curso } & Medicina & 10 & 27,0 \\
\hline & Medicina e outros cursos da saúde & 2 & 5,4 \\
\hline & Enfermagem & 6 & 16,2 \\
\hline & Enfermagem e outros cursos da saúde & 2 & 5,4 \\
\hline & Enfermagem; Obstetrícia; Educação & 1 & 2,7 \\
\hline & Educação & 1 & 2,7 \\
\hline & Odontologia & 1 & 2,7 \\
\hline & Não identificado & 14 & 37,8 \\
\hline
\end{tabular}

*Índice de Desenvolvimento Humano, segundo o relatório do Programa das Nações Unidas para o Desenvolvimento, 2014.

mínio mastery (estar sob o controle dos eventos da vida e não ser fatalista). Os fatores inseridos na categoria Relacionais (6) foram apoio social elevado, ter recebido apoio dos pais, ter habilidades comunicacionais e engajamento social.

\section{Discussão}

Nesta revisão, identificou-se prevalências e fatores de risco e proteção para sofrimento psíquico entre universitários nos últimos dez anos. Dez artigos foram identificados em 2014, sendo seis ${ }^{7-11,50}$ brasileiros que investigaram TMC utilizando o SRQ20 e o GHQ-12 ${ }^{38}$. Destaca-se a constância mantida no número de publicações ao longo desses anos, com exceção de 2013. 


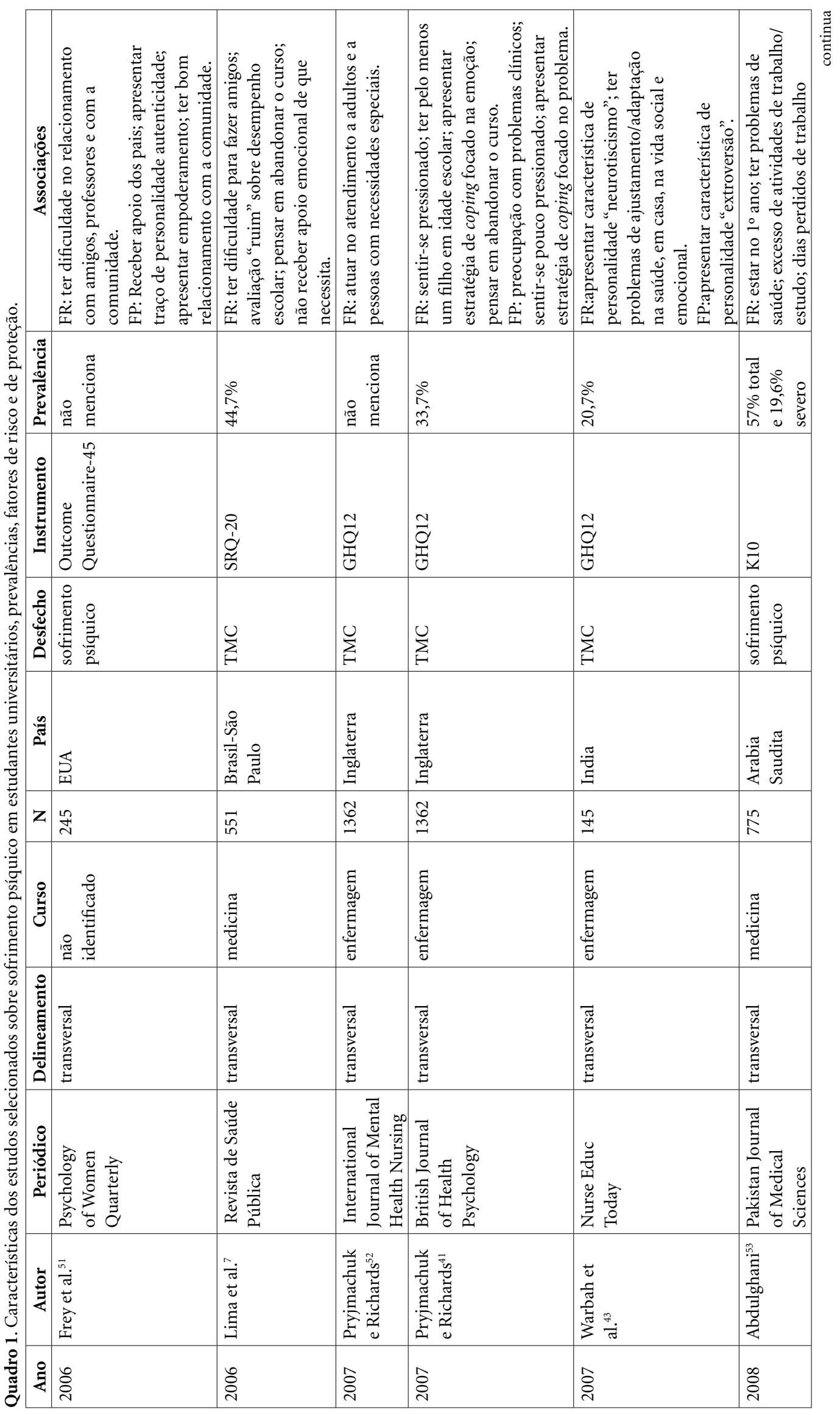




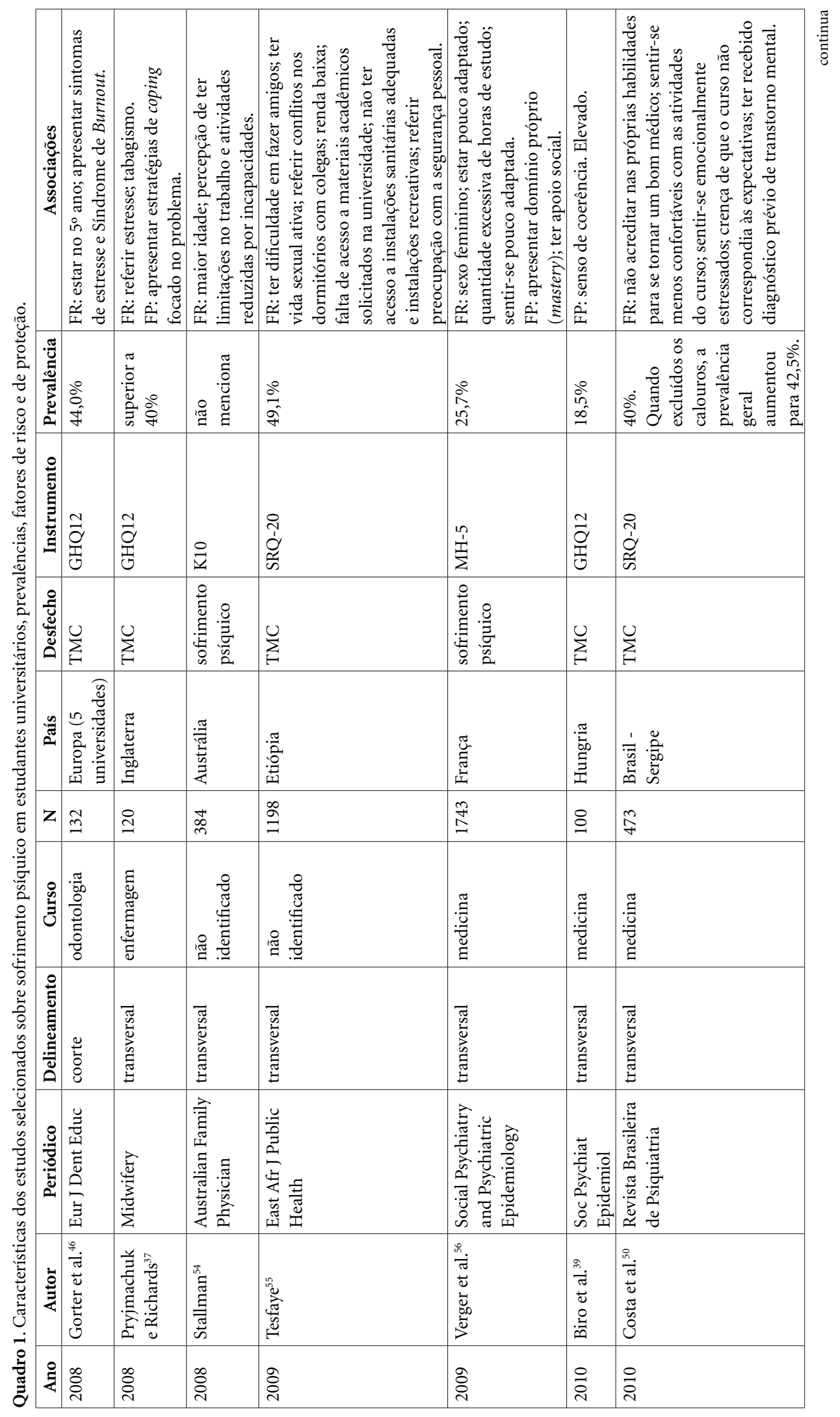




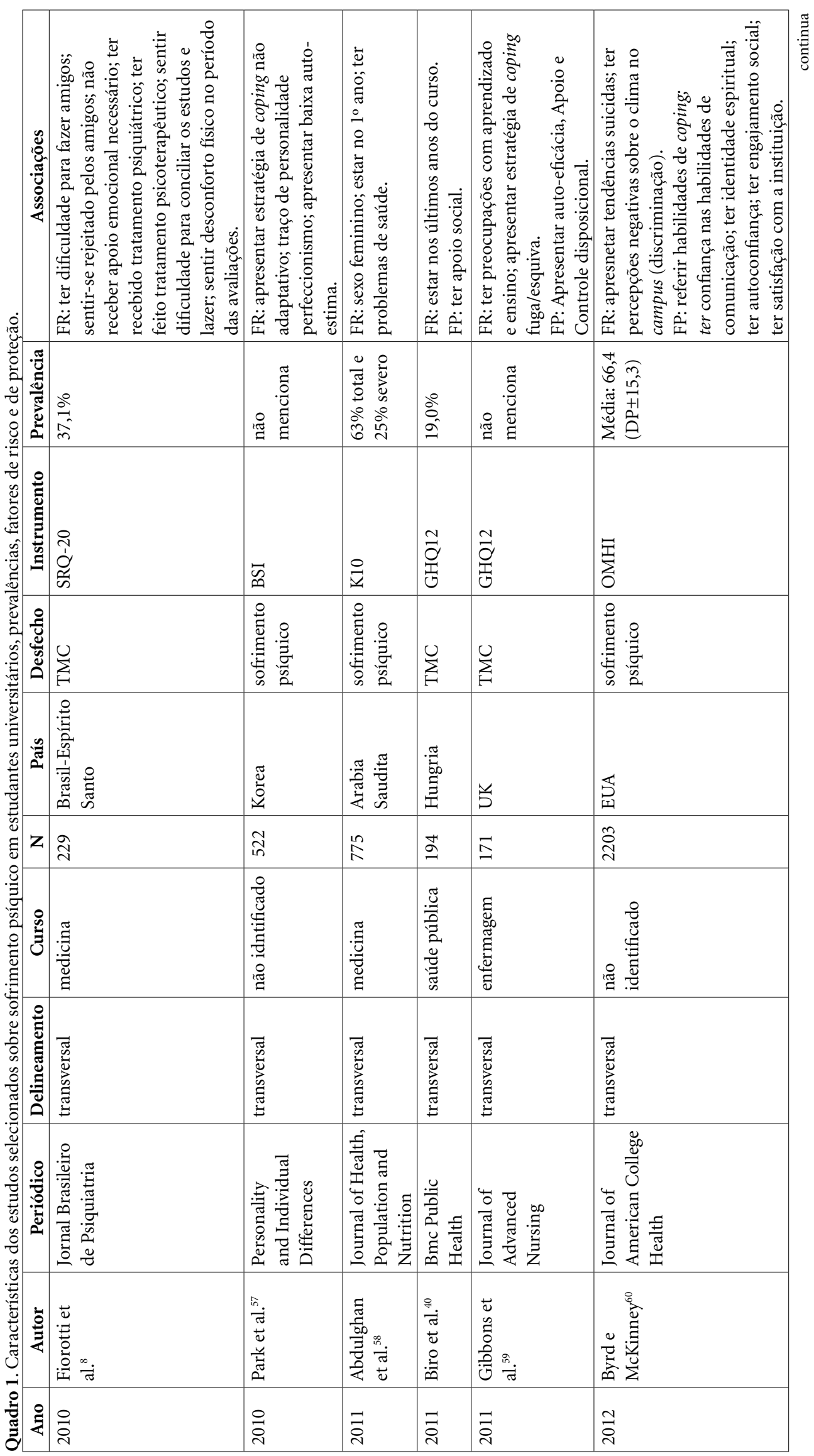




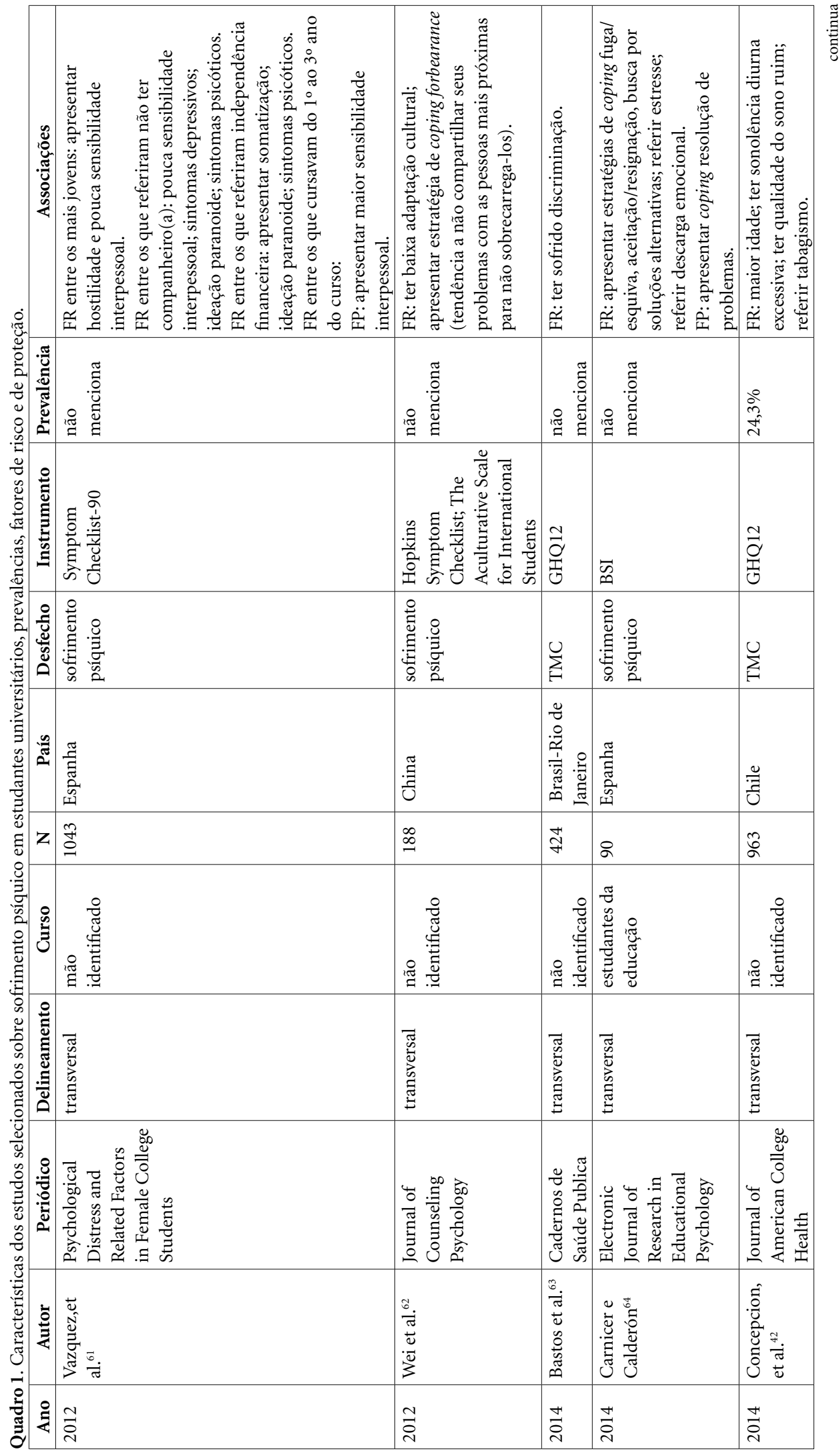




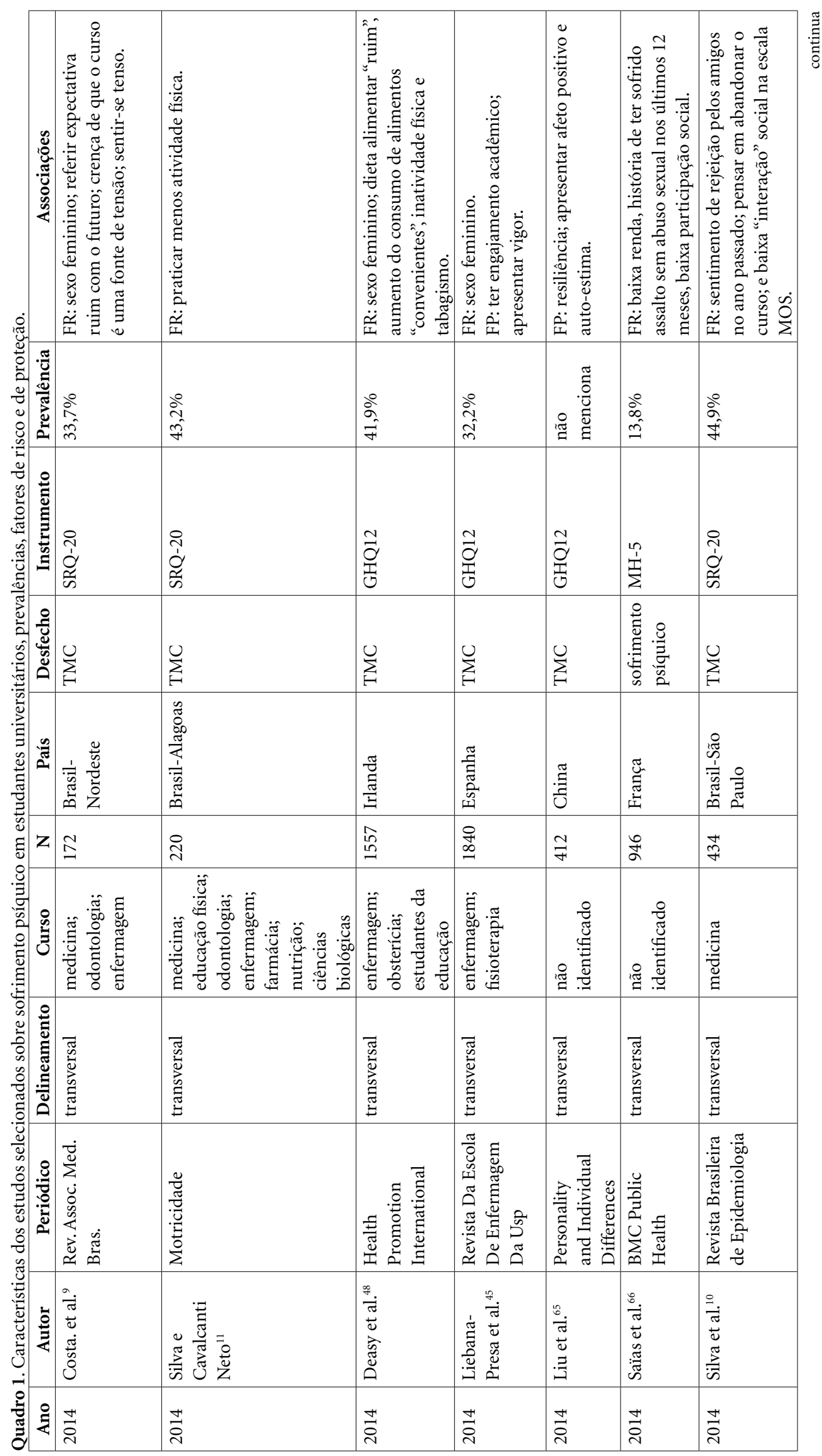




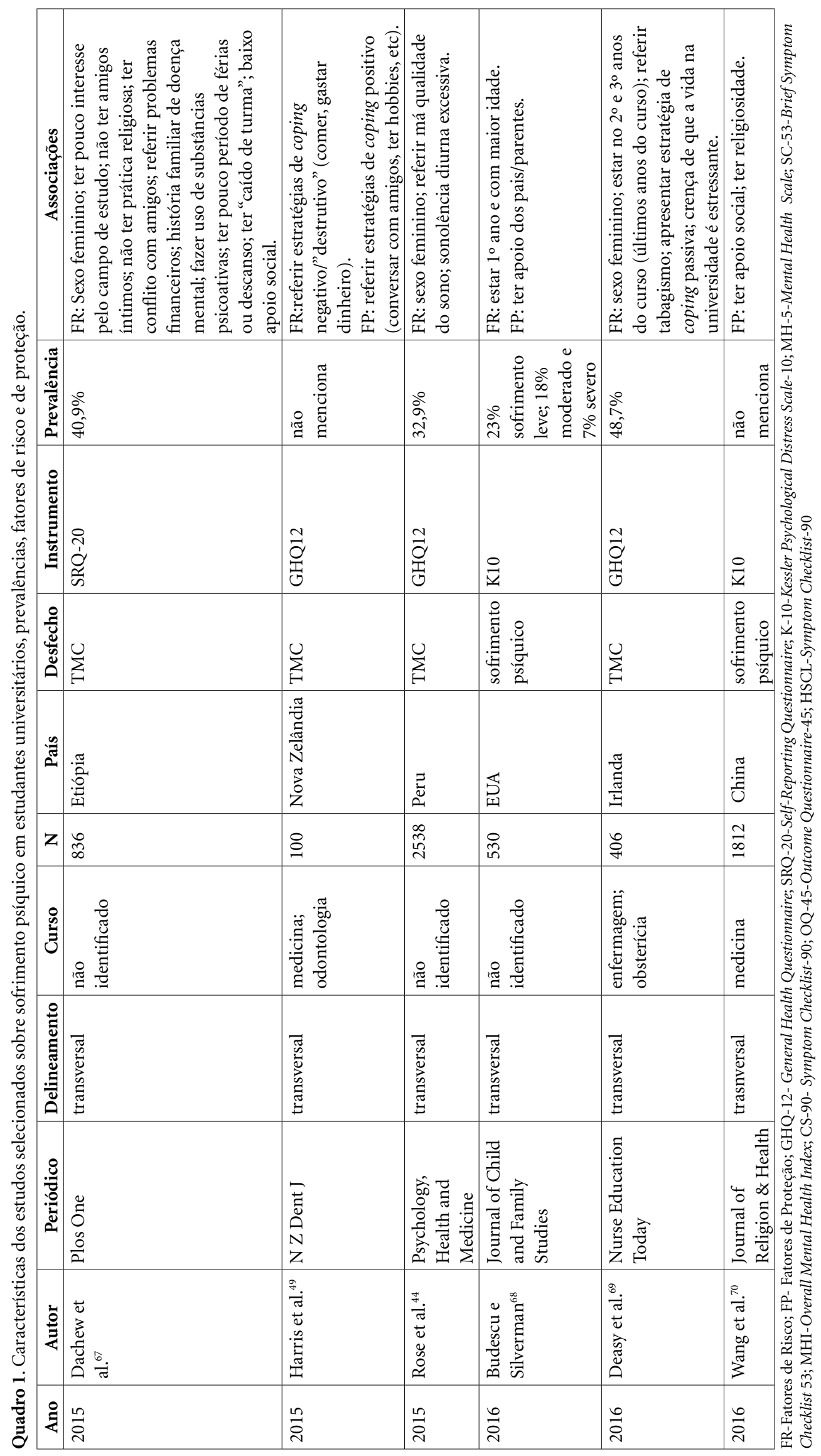


Tabela 2. Frequência de categorias e variáveis de risco associadas ao sofrimento psíquico dos estudantes universitários identificadas nos artigos $(\mathrm{n}=37)$.

\begin{tabular}{|c|c|c|}
\hline \multicolumn{3}{|c|}{ Fatores de Risco } \\
\hline Categorias & Variáveis & $\mathbf{n}$ \\
\hline Socioemográficas & Sexo (feminino) & 8 \\
\hline \multirow[t]{6}{*}{$(\mathrm{n}=18)$} & Idade (Maior) & 3 \\
\hline & Renda (baixa) & 3 \\
\hline & Religião (Não) & 1 \\
\hline & Residência sem saneamento básico & 1 \\
\hline & Ter filho em idade escolar (Sim) & 1 \\
\hline & Ter companheiro (a) (Sim) & 1 \\
\hline Saúde & Tabagismo (Sim) & 4 \\
\hline \multirow[t]{15}{*}{$(\mathrm{n}=22)$} & Problemas de saúde (Sim) & 2 \\
\hline & Atividade física (Não) & 2 \\
\hline & Referir estresse (Sim) & 2 \\
\hline & Dieta alimentar (Ruim) & 1 \\
\hline & Sonolência diurna excessiva (Sim) & 1 \\
\hline & Percepção de limitações/incapacidades (Sim) & 1 \\
\hline & Qualidade do sono ruim (Sim) & 1 \\
\hline & Sentir-se tenso (Sim) & 1 \\
\hline & Dias perdidos de trabalho por saúde (Sim) & 1 \\
\hline & Ter recebido diagnóstico de TM (Sim) & 1 \\
\hline & Uso de substância (Khat chewing) (Sim) & 1 \\
\hline & Ter recebido tratamento psicológico (Sim) & 1 \\
\hline & Histórico familiar de Transtorno Mental (Sim) & 1 \\
\hline & Tendência suicida (Sim) & 1 \\
\hline & Vida sexual ativa (Sim) & 1 \\
\hline Relacionais & Dificuldades no relacionamento com amigos (Sim) & 5 \\
\hline \multirow[t]{5}{*}{$(\mathrm{n}=15)$} & Adaptação à vida acadêmica ou outros aspectos da vida (Não) & 3 \\
\hline & Sentir-se rejeitado pelos amigos (Sim) & 2 \\
\hline & Apoio social (Não) & 2 \\
\hline & Receber apoio emocional necessário (Não) & 2 \\
\hline & Dificuldade em relacionar-se com as pessoas (Sim) & 1 \\
\hline Acadêmicas & Série do curso (Primeiras séries) & 3 \\
\hline \multirow[t]{12}{*}{$(\mathrm{n}=24)$} & Série do curso (Últimas séries) & 2 \\
\hline & Excesso de horas de estudo/Dificuldade conciliar estudo x lazer (Sim) & 3 \\
\hline & Ter pensado em abandonar o curso (Sim) & 3 \\
\hline & Curso como fonte de tensão ou estresse (Sim) & 2 \\
\hline & Expectativa com o futuro profissional (Ruim) & 2 \\
\hline & Satisfação com o curso ou interesse pelo curso (Não) & 2 \\
\hline & Desconforto com as atividades do curso ou durante as avaliações (Sim) & 2 \\
\hline & Preocupações com aprendizado e ensino (Sim) & 1 \\
\hline & Disponibilidade de materiais para o curso (Pouca) & 1 \\
\hline & Auto avaliação do desempenho acadêmico (Ruim) & 1 \\
\hline & Ter "caído de turma" (Sim) & 1 \\
\hline & Atuação: adultos e necessidades especiais & 1 \\
\hline
\end{tabular}

Observou-se, também, variabilidade dos desfechos investigados, bem como dos instrumentos utilizados, fato já identificado em revisão sistemática que incluiu publicações entre 1980 e 2005 sobre sofrimento psíquico em estudantes do curso de medicina ${ }^{71}$.

Dos 37 estudos avaliados, apenas um não apresentou delineamento transversal, provavel- 
Tabela 2. Frequência de categorias e variáveis de risco associadas ao sofrimento psíquico dos estudantes universitários identificadas nos artigos $(\mathrm{n}=37)$.

\begin{tabular}{llc}
\hline \multicolumn{1}{c}{ Categorias } & \multicolumn{1}{c}{ Fatores de Risco } & \multicolumn{1}{c}{ Variáveis } \\
\hline Psicológicas & $\begin{array}{l}\text { Coping fuga/esquiva, aceitação/resignação, busca por soluções } \\
\text { alternativas, descarga emocional (Escores elevados) }\end{array}$ & 1 \\
& Coping fuga-esquiva (Escores elevados) & \\
& Coping forbearance (Escores elevados) & 1 \\
& Coping passivo (Escores elevados) & 1 \\
& Coping focado na emoção (Escores elevados) & 1 \\
& Referir Coping negativo ou destrutivo (comer, gastar dinheiro) (Sim) & 1 \\
& Coping não adaptativo (Escores elevados) & 1 \\
& Neuroticismo (Escores elevados) & 1 \\
Social/Violência & Perfeccionismo e baixa auto-estima (Escores elevados) & 1 \\
$(\mathrm{n}=4)$ & Percepção de discriminação (raça, idade, e classe) (Sim) & 1 \\
& Percepção de clima ruim na universidade em relação à discriminação & 1 \\
& Preocupação com a segurança pessoal (Sim) & 1 \\
& Ter sofrido agressão (Sim) & 1 \\
\hline
\end{tabular}

Tabela 3. Frequência de categorias e variáveis de risco associadas a sofrimento psíquico por artigo $(\mathrm{n}=37)$.

\begin{tabular}{lll}
\hline \multicolumn{1}{c}{ Categorias } & \multicolumn{1}{c}{ Fatores de Proteção } & \multicolumn{1}{c}{ Variáveis } \\
\hline Demográficas $(\mathrm{n}=2)$ & Ter religião (Sim) & 2 \\
Relacionais $(\mathrm{n}=6)$ & Apoio social (Alto) & 3 \\
& Receber apoio dos pais (Sim) & 1 \\
& Ter habilidades de comunicação (Sim) & 1 \\
& Apresentar engajamento social (Sim) & 1 \\
Acadêmicas $(\mathrm{n}=2)$ & Apresentar engajamento acadêmico (Sim) & 1 \\
& Sentir-se pressionado (Pouco) & 1 \\
Psicológicas $(\mathrm{n}=12)$ & Coping focado no problema (Escores elevados) & 2 \\
& Apresentar autenticidade e empoderamento (Escores elevados) & 1 \\
& Coping resolução de problemas (Escores elevados) & 1 \\
& Referir habilidades de coping, em geral; e ter auto-confiança (Escores elevados) & 1 \\
& Coping positivo (falar com amigos/hobbies) (Sim) & 1 \\
& Apresentar senso de coerência (Escores elevados) & 1 \\
& Apresentar auto-eficácia e controle disposicional (Escores elevados) & 1 \\
& Apresentar vigor (Escores elevados) & 1 \\
& Apresentar afeto positivo; auto-estima; e resiliência (Escores elevados) & 1 \\
& Apresentar extroversão (Escores elevados) & 1 \\
& Apresentar domínio próprio mastery (Escores elevados) & 1 \\
\hline
\end{tabular}

mente devido à dificuldade de se realizarem estudos longitudinais com essa população ${ }^{56,66}$. Os estudos transversais analisados utilizaram medidas de força que evidenciaram associações entre as características e os aspectos individuais, inter -relacionais e institucionais ${ }^{60}$ dos estudantes ao sofrimento psíquico; no entanto, características próprias do desenho transversal não permitem a identificação de causalidade.

Do total da amostra, 16 investigaram TMC com o GHQ-12 $39-69,52,59,65,63$ e oito com o SRQ-20 $0^{7-}$ 11,50-55. Observou-se maior variação das prevalências obtidas pelo GHQ-12, comparativamente às obtidas pelo SRQ-20, aplicado predominan- 
temente em alunos de universidades brasileiras, chamando atenção para a diferença na amplitude de variação das prevalências desses instrumentos (Quadro 1). Apesar de ambos apresentarem melhores propriedades psicométricas, comparativamente ao K-10, K-6 e ao Patient Health Questionnaire, segundo Patel et al. ${ }^{38}$, o GHQ-20 apresentou melhores índices do likelihood ratio refletindo maior sensibilidade e especificidade do teste. Além disso, sugere-se que a amplitude obtida pelo GHQ-12 nas prevalências ocorreu por ter sido aplicado em vários países, com diferenças culturais, e o SRQ-20 ter sido predominantemente utilizado em estudos brasileiros.

No Brasil, as prevalências de TMC em universitários foram superiores ao identificado na população geral ${ }^{1}$ e entre adolescentes ${ }^{2}$, mas inferiores às obtidas em usuários da Estratégia da Saúde da Família ${ }^{3}$. Destaca-se que a maior prevalência de casos de TMC $(49,1 \%)^{55}$ foi encontrada em universitários da Etiópia, superior às identificadas entre alunos da saúde de países desenvolvidos e em desenvolvimento ${ }^{55}$. A Etiópia é um país onde grande parte da população vive em condições precárias e com preocupação constante em relação à segurança pessoal ${ }^{55}$.

Elevadas prevalências de sofrimento psíquico, avaliado pelo K-10, foram identificadas em estudantes na Arábia Saudita ${ }^{58,53}$. Fatores econômicos podem predispor os alunos a maior sofrimento ${ }^{1}$. TMC tem sido apontado como mais prevalente em pessoas com baixa renda, com baixos níveis de educação e residentes em países subdesenvolvidos ou em desenvolvimento ${ }^{1,52}$. Neste estudo, as menores prevalências identificadas (13,8\%, $18,5 \%$ e $19,0 \%$ ) foram em pesquisas realizadas na França $a^{53,56}$ e na Hungria ${ }^{39,40}$, países com elevado IDH. Ambos os estudos realizados na Hungria tiveram uma amostra menor que as de outros estudos aqui descritos e a pesquisa desenvolvida na França obteve os dados por meio de entrevistas telefônicas, diferenças metodológicas que devem ser consideradas.

Dado que elevadas prevalências de sofrimento psíquico têm sido obtidas entre universitários, em diversos países, investigar características desses jovens e aspectos associados pode favorecer o planejamento de estratégias de prevenção e de cuidado com essa população e a diminuição da evasão escolar ${ }^{60}$.

\section{Características sociodemográficas}

Identificou-se que sexo feminino ${ }^{9,44,45,48,69-67}$, maior idade $^{42,68,54}$ e baixa renda ${ }^{67,55,66}$ foram as características sociodemográficas mais associadas a sofrimento psíquico entre universitários, características também obtidas em revisão sistemática $^{71}$. Esses dados reforçam que condições socioeconômicas desfavoráveis, acentuadas pela ainda vigente discriminação de gênero em muitas culturas, podem contribuir para o sofrimento psíquico. Fatores genéticos e fisiológicos podem influenciar padrões de morbidade e mortalidade de homens e mulheres, porém sabe-se que distintos valores, processos de socialização e coping são observados e podem determinar o adoecimento. Além disso, possivelmente as mulheres tenham maior facilidade de identificar e relatar sintomas, buscando ajuda ou apoio social, enquanto homens busquem alívio para o sofrimento através do uso de substâncias, como o álcool ${ }^{72}$.

Nesta revisão, os dois estudos realizados na Etiópia identificaram como fatores de risco para sofrimento psíquico não ter religião ${ }^{67}$ e residir em local sem saneamento básico ${ }^{55}$. Duas pesquisas, uma nos EUA $(\mathrm{n}=2203)^{60}$ e outra com estudantes de uma universidade chinesa $(n=1812)^{70}$, encontraram que religiosidade/identidade espiritual foi fator de proteção, supondo que aspectos culturais exercem influência no desenvolvimento de $\mathrm{TMC}^{73}$. Ter filhos em idade escolar ${ }^{52}$ e não possuir companheiro $(a)^{61}$ foram indicados como risco para sofrimento psíquico nesta revisão, sugerindo que estudantes com dificuldades financeiras e dependentes possuem maior chance de apresentar sofrimento ${ }^{67,66}$.

\section{Condições de saúde}

Mais da metade dos estudos apresentou associação entre sofrimento psíquico e aspectos da saúde de graduandos. Estes podem comportar-se como modificadoras e/ou mediadoras, acentuando ou atenuando o sofrimento ${ }^{58,53}$. Identificou-se que tabagismo foi fator de risco para TMC em quatro pesquisas ${ }^{40,42,47,69}$. Na Etiópia ${ }^{67}$, alunos que utilizavam substância psicoativa Khat Chewing, com ação estimulante, apresentaram maior prevalência de sofrimento psíquico. Considera-se que provavelmente o uso de substâncias ocorra para atenuar o estresse ${ }^{48}$, sendo uma estratégia de coping, do tipo fuga/esquiva que, dependendo da intensidade e frequência, pode acarretar prejuízos aos indivíduos ${ }^{47,67}$. Além disso, foram identificados como fatores de risco para TMC não praticar atividades físicas e apresentar dieta alimentar inadequada, mais frequentes em estudantes dos últimos semestres ${ }^{11}$. A prática de atividades físicas regulares e a alimentação saudável 
podem exercer função de proteção à saúde dos indivíduos reduzindo os níveis de estresse ${ }^{48}$. Sonolência, má qualidade do sono ${ }^{42,44}$, estresse ${ }^{46,47}$ e tensão ${ }^{9}$ também associaram-se significativamente à sofrimento psíquico entre universitários. Essas condições são frequentes, considerando-se a rotina de estudos imposta para atingir as exigências acadêmicas e da grade curricular dos cur$\operatorname{sos}^{9,15-18,28,31,71}$.

Nesta revisão, identificou-se que estresse e Síndrome de Burnout (SB) associaram-se à TMC em alunos de odontologia na Europa. Em análise de fatores de mediação, observou-se efeito direto do estresse na saúde física dos estudantes e indireto na saúde mental, tendo sido este mediado por $\mathrm{SB}^{46}$. Analisar os "caminhos" que levam ao sofrimento psíquico pode contribuir no planejamento de estratégias para minimizar os problemas de saúde ${ }^{53,54}$. O estresse pode "passar despercebido" pelos indivíduos ou ser considerado "normal"; porém, quando elevado pode-se observar o sofrimento psíquico ${ }^{46}$. Revisão sobre estresse em universitários de odontologia corrobora com os dados aqui obtidos ${ }^{74}$.

Identificou-se que indivíduos que receberam tratamento psicológico/psiquiátrico ${ }^{8}$, com histórico familiar ${ }^{67,54}$ e pessoal ${ }^{53}$ de transtornos mentais, apresentaram maior prevalência de TMC. Essa associação envolve tanto a presença de fatores hereditários/genéticos como a sobrecarga emocional decorrente dos cuidados para com o familiar doente ${ }^{67}$. $\mathrm{O}$ sofrimento psíquico, também, associa-se à tendência suicida entre estudantes ${ }^{60}$, avaliada por instrumento específico (MHI). Suicidalidade (ideação, planejamento, tentativas de suicídio), em especial entre alunos de medicina, é uma preocupação crescente associada à depressão ${ }^{75}$.

\section{Características relacionais}

Constatou-se que as relações estabelecidas pelos estudantes podem ter papel de risco ou proteção à saúde mental. As características associadas a sofrimento psíquico foram: ter dificuldade em fazer amigos ${ }^{7,8,55,51}$, relações conflitivas ${ }^{67}$, sentir-se rejeitado ${ }^{8,10}$, não receber apoio emocional necessário $^{7,8}$, dificuldade de adaptação ${ }^{43,56}$. Percepção de pouco apoio social associou-se em dois estudos à prevalência mais elevada de $\mathrm{TMC}^{10,67}$. Silva et al. ${ }^{10}$ identificaram que o domínio interação social positiva insuficiente (habilidade de se divertir/relaxar), avaliada pela Escala de Apoio Social, apresentou-se como fator de risco para TMC entre estudantes de medicina. Infere-se que alunos que não dispõe de amigos para compartilhar momentos sociais, apresentam maior isolamento e sofrimento. Assim, ter apoio social, possuir habilidades sociais e engajar-se em atividades de lazer são apontadas como variáveis de proteção para a saúde mental dos alunos.

\section{Percepção da vida acadêmica}

Aspectos da vida acadêmica são potenciais fatores de risco para sofrimento psíquico dos estudantes, em especial, da área da saúde $\mathrm{e}^{16-18,20,76}$. Nesta revisão, essas variáveis foram as mais frequentemente investigadas (Tabelas 2 e 3). Verificou-se que alunos das primeiras e últimas séries foram os que mais apresentaram sofrimento. Estudos realizados com universitários na Arábia Saudita ${ }^{58,53}$ mostraram maior chance (odds ratio) de sofrimento entre alunos do primeiro ano de medicina, e um realizado na Hungria ${ }^{40}$ identificou que estar nos últimos anos foi um fator de risco para $\mathrm{TMC}^{46}$. Diferentemente, pesquisa com alunos de enfermagem e formação de obstetrizes observou aumento progressivo da prevalência de TMC ao longo das séries ${ }^{58}$.

Pode-se considerar que, no início dos cursos, os jovens deparam-se com mudanças na vida pessoal/social (mudança de cidade, morarem sozinhos) e universitária (novos métodos de estudo, extensa grade curricular). Nos últimos anos, o contato com pacientes, proximidade com o sofrimento e a morte, preocupação com o aprendizado de procedimentos clínicos tendem a desencadear ou acentuar o estresse entre os estudantes ${ }^{4,15,56,71}$. Ao contrário, indivíduos que possuem apoio social tendem a apresentar melhor percepção do desempenho acadêmico, independentemente da série, favorecendo menor sofrimento ${ }^{68}$.

Identificou-se que alunos mais engajados nas atividades do curso ${ }^{45}$ e que se sentiam pouco pressionados $^{43}$ tiveram melhores resultados em sua saúde mental. A percepção dos alunos sobre sua vivência na universidade pode influenciar a sensação de bem estar. Estudantes que haviam pensado em abandonar o curso ${ }^{7,41}$, percebiam-no como fonte de estresse $e^{9,69}$, desconforto ${ }^{50}$, em especial, durante as avaliações ${ }^{8}$, insatisfação com o curso $^{50}$ e baixas expectativas com o futuro profissional ${ }^{9}$ apresentaram elevado sofrimento psíquico. Revisão sistemática ${ }^{74}$ também identificou que fatores acadêmicos foram os mais referidos como estressores entre alunos de odontologia, tanto na fase pré-clínica como clínica. 


\section{Características psicológicas}

Revisão realizada por Dyrbye et al. ${ }^{71}$ mostrou que autoconhecimento e sensação de cumprimento dos deveres foram variáveis de proteção à saúde mental dos estudantes, enquanto perfeccionismo e supressão da raiva associaram-se a maiores escores de sofrimento. Nesta revisão, características psicológicas foram frequentemente identificadas como proteção para sofrimento psíquico, como senso de coerência (orientação que expressa sentimento de confiança ${ }^{39}$, autenticidade e empoderamento (força pessoal que emerge da relação com o outro) ${ }^{51}$, autoeficácia (crença sobre as próprias capacidades) e controle disposicional (avaliação cognitiva em uma situação) ${ }^{59}$, autoestima elevada e afeto positivo (sentimento de engajamento prazeroso com o ambiente) ${ }^{65}$, maior vigor (energia durante o aprendizado) ${ }^{45}$, elevada resiliência ${ }^{65}$, extroversão (cheio de energia, bem relacionado ${ }^{43}$ e escores elevados para o domínio mastery (controle dos eventos da vida), do Eisenck Personality Questionnair ${ }^{56}$. Ao contrário, alunos que apresentaram escores para neuroticismo (ansiedade, medo, preocupação, frustração $)^{43}$, perfeccionismo e baixa autoestima ${ }^{57}$ tiveram maior risco de apresentar sofrimento psíquico. Assim, salienta-se que esses resultados indicam que algumas características individuais podem exercer influência nas estratégias de coping, reduzindo a possibilidade de sofrimento ${ }^{77}$.

Estratégias de coping também foram identificadas como risco para sofrimento em universitários, como: coping focalizado na emoção ${ }^{41}$, fuga/ esquiva ${ }^{59,64}$, coping passivo ${ }^{69}$, referir estratégias de coping negativas/destrutivas (comer muito, gastar dinheiro $)^{49}$, coping não adaptativo ${ }^{57}$, e coping forbearance (não compartilhar problemas, omissão $)^{62}$. Porém, estratégias de coping ativas como focalizadas no problema ${ }^{41,47}$, resolução de problemas $^{64}$, coping positivo ${ }^{49}$ (falar com amigos, lazer), ou referir ter habilidades de enfrentamento ${ }^{60}$ associaram-se a menor sofrimento.

Estratégias de coping podem ser definidas em duas categorias funcionais (problema e emoção), que podem ser complementares. A primeira inclui esforços para identificar o problema e buscar recursos para controlar o estressor, enquanto as focalizadas na emoção incluem comportamentos para suportar o impacto do evento ${ }^{77,78}$. Estratégias de coping ${ }^{77}$ incluem processo adaptativo de aprendizado e resultados em saúde ${ }^{77}$.

A resiliência também é uma característica que pode ser considerada como protetora à saú- de dos indivíduos ${ }^{79}$, avaliada em estudos com diferentes populações com elevada carga de estresse $^{79}$. Dentre estudos empíricos, uma revisão identificou que apenas cinco tiveram como foco

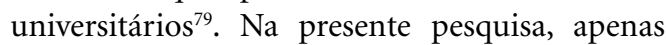
um estudo investigou a associação de resiliência e TMC entre estudantes ${ }^{50}$, mostrando que resiliência influenciou positivamente a satisfação com a vida. Em outro estudo, constatou-se que estratégias de coping no problema foram preditivas para alta resiliência ${ }^{80}$. Pode-se inferir que características psicológicas influenciam positivamente padrões de resiliência impedindo a ocorrência de sofrimento psíquico.

\section{Variáveis sociais/violência}

Esta revisão identificou quatro publicações sobre saúde mental e discriminação em universitários. Identificou-se como fatores de risco para sofrimento: discriminação (racial, idade, classe, orientação sexual ${ }^{60,63}$, ter preocupação com a segurança pessoal e ter sofrido agressã $0^{66}$.

Bastos et al ${ }^{63}$, identificaram associação entre experiências de discriminação e TMC, utilizando questionário desenvolvido e adaptado para a população brasileira. Resultados apontaram que $23 \%$ dos alunos referiram ter sofrido discriminação, tendo sido mais frequente entre as mulheres, "quotistas", com baixa renda e entre negros/ pardos. Todos os tipos de discriminação associaram-se significativamente à TMC; além disso, os estudantes que referiram discriminação foram 14 vezes mais propensos a apresentar sofrimento $^{63}$. Discriminação racial e de classe social entre universitários reflete a realidade do contexto sociocultural brasileiro, em que aspectos relativos às desigualdades sociais e iniquidades em saúde encontram-se atrelados a essa questão ${ }^{56}$. Byrd e McKinney ${ }^{60}$ também identificaram que percepção de discriminação (racial, sexo e orientação sexual) e insatisfação com a instituição estiveram associadas à sofrimento psíquico.

Embora não identificado nesta revisão, o trote, prática comum nas universidades brasileiras, principalmente em escolas médicas ${ }^{81}$, é uma violência escolar que requer atenção por envolver agressões, humilhações e o uso problemático de substâncias, como o álcool ${ }^{82}$. A literatura aponta que situações de violência, viver sob a preocupação com a segurança pessoal, são preditores importantes para sofrimento e piores índices de saúde entre os indivíduos ${ }^{83,84}$. 


\section{Considerações finais}

Esta revisão possibilitou traçar um panorama sobre o sofrimento psíquico/transtornos mentais comuns (TMC) entre estudantes universitários, focalizando-se especialmente os fatores de risco e proteção a eles associados. Os diferentes instrumentos de screening, com boas qualidades psicométricas $^{38}$, que têm sido utilizados nos estudos, possibilitam análise comparativa de resultados entre estudantes de diferentes países e regiões. Constatou-se que pesquisas com este objetivo têm sido realizadas em diversos países, com maior frequência nos desenvolvidos e com universitários de cursos da saúde. Os resultados, no Brasil, em geral, demonstraram semelhanças em relação às prevalências de TMC, sendo estas mais elevadas que as identificadas na população geral, porém inferiores às obtidas em estudo com usuários da Atenção Primária à Saúde. Diferenças socioculturais identificadas entre as populações estudadas favoreceram a compreensão desses dados.

A análise das características que mais associaram-se ao sofrimento psíquico entre universitários evidenciou predominância dos aspectos acadêmicos (séries do curso e percepção negativa do ambiente) como fatores de risco. Alguns aspectos da saúde dos universitários (hábitos prejudiciais à saúde e problemas de saúde) associaram-se à presença de TMC e, por outro lado, indivíduos com apoio social apresentaram menor sofrimento psíquico. A maioria dos estudos identificados nesta revisão apresentaram delineamento transversal, investigando os desfechos em um único momento, tornando mais difícil indicar a existência de relação causal entre as variáveis investigadas. Assim, estudos prospectivos devem ser desenvolvidos acompanhando os estudantes ao longo das séries dos cursos.

Pode-se concluir que características da vida acadêmica e relacionais foram as que mais frequentemente associaram-se à presença de sofrimento psíquico entre universitários, fatores passíveis de modificação, mostrando a importância do planejamento de intervenções favorecendo o bem estar dos alunos e vivências mais positivas no ambiente educacional. Violência escolar, trote, bullying, discriminação social entre universitários, estruturas pedagógicas e curriculares dos cursos são aspectos que devem ser mais profundamente pesquisados, buscando-se compreender sua influência sobre os jovens e seus efeitos negativos na saúde dos indivíduos.

\section{Colaboradores}

KM Graner e ATAR Cerqueira trabalharam igualmente em todas as etapas deste estudo, incluindo planejamento, realização da coleta dos dados, análise dos dados, descrição dos resultados, elaboração da discussão e redação final.

\section{Agradecimentos}

À Fundação de Amparo à Pesquisa do Estado de São Paulo, FAPESP, pela bolsa de doutorado concedida à Karen Mendes Graner. 


\section{Referências}

1. Schimidt MI, Duncan BB, Silva GA, Menezes AM, Monteiro CA, Barreto SM, Chor D, Menezes PR. Doenças crônicas não transmissíveis no Brasil: carga e desafios atuais. Lancet 2011; 9:61-74.

2. Lopes CS, Abreu GA, Santos DF, Menezes PR, Carvalho KMB, Cunha CF, Vasconcellos MTL, Bloch KV, Szklo M. ERICA: prevalência de transtornos mentais comuns em adolescentes brasileiros. Rev Saude Publica 2016; 50(Supl. 1):1-14.

3. Santos E, Siqueira MM. Prevalência dos transtornos mentais na população adulta brasileira: uma revisão sistemática de 1997 a 2009. J Bras Psiquiatr 2010; 59(3):238-246.

4. Fagundes VLD, Ludermir AB. Common mental disorders among health care students. Rev Bras Psiquiatr 2005; 27(3):194-200.

5. Hidalgo MPL, Ponte TS, Carvalho CG, Pedrotti MR, Nunes PV, Souza CM, Zanette CB, Voltolini S, Chaves MLF. Association between mental health screening by self-report questionnaire and insomnia in medical students. Arch Neuropsyquiatry 2001; 59(2-A):180-185.

6. Volcan SMA, Sousa PLR, Mari JJ, Horta BL. Relação entre bem-estar espiritual e transtornos psiquiátricos menores: estudo transversal. Rev Saude Publica 2003; 37(4):440-445.

7. Lima MCP, Domingues MS, Ramos-Cerqueira ATA. Prevalência e fatores de risco para transtornos mentais comuns entre estudantes de Medicina. Rev Saude Publica 2006; 40(6):1035-1041.

8. Fiorotti KP, Rossoni RR, Borges, LH, Miranda AE. Transtornos mentais comuns entre os estudantes do curso de medicina: prevalência e fatores associados. $J$ Bras Psiquiatr 2010; 59(1):17-23.

9. Costa EFO, Rocha MMV, Santos ATRA, Melo EV, Martins LAN, Andrade TM. Common mental disorders and associated factors among final-year healthcare students. Rev Assoc Med Bras 2014; 60(6):525-530.

10. Silva AG, Ramos-Cerqueira ATA, Lima MCP. Social support and common mental disorder among medical students. Rev Bras Epidemiol 2014; 17(1):229-242.

11. Silva PO, Cavalcante-Neto JP. Associação entre níveis de atividade física e transtorno mental comum em estudantes universitários. Motricidade 2014; 10(1):49-59.

12. Goldberg D, Huxley P. Common Mental Disorders-A bio-social model. Tavistock: Routledge; 1993.

13. American Psychiatric Association. DSM-V. $5^{\mathrm{a}}$ ed. Porto Alegre: Artmed; 2014.

14. Organização Mundial da Saúde (OMS). Classificação de Transtornos Mentais e de Comportamento da CID-10. Trad. Dorgival Caetano. Porto Alegre: Artes Médicas; 1997.

15. Nogueira-Martins LS. Saúde Mental dos profissionais da saúde Rev Bras Med Trab 2003; 1(1):56-68.

16. Herrera C, Pacheco J, Rosso F, Cisterna C, Aichele D, Becker S, Padilla O, Riquelme A. Evaluación del ambiente educacional pre-clínico en seis Escuelas de Medicina. Rev Med Chile 2010; 138:677-684.

17. Thurber CA, Walton EA. Homesickness and adjustment in university students. J Am Col Health 2012; 60(5):415-419.

18. Alzahem AM, Van der Molen HT, Boer BJ. Effect of year of study on stress levels in male undergraduate dental students. Adv Med Educ Pract 2013; 4:217-222.
19. Divaris K, Mafla AC, Villa-Torres L, Sánchez-Molina M, Gallego-Gómez CL, Vélez-Jaramillo LF, TamayoCardona JA, Pérez-Cepeda D, Vergara-Mercado ML, Simancas-Pallares MA. Polychronopoulo Psychological distress and its correlates among dental students: a survey of 17 Colombian dental schools. BMC Med Edu 2013; 13:91.

20. Feodrippe IALO, Brandão MCF, Valente ITCO. Qualidade de vida de estudantes de medicina: uma revisão Rev Bras Ed Med 2013; 37(3):418-428.

21. Maia J, Willians LCA. Fatores de risco e fatores de proteção ao desenvolvimento infantil: uma revisão da área. Temas em Psicologia 2005; 13(2):91-103.

22. Sapienza G, Pedromônico MRM. Risco, proteção e resiliência no desenvolvimento da criança e do adolescente. Psicol Est 2005; 10(2):209-216.

23. Buss PM, Pellegrini-Filho A. A saúde e seus determinantes sociais. Physis 2007; 17(1):77-93.

24. Almeida-Filho N, Coutinho D. Causalidade, contingência, complexidade: o futuro do conceito de risco. Physis 2007; 17(1):95-137.

25. Souza FGM, Menezes MGC. Estresse nos estudantes de Medicina da Universidade Federal do Ceará. Rev Bras Edu Méd 2005; 29(2):91-96.

26. Madhan B, Rajpurohit AS, Gayathri H. Mental Health of Postgraduate Orthodontic Students in India: A Multi-Institution Survey. J Dent Educ 2012; 76(2):200-209.

27. Amaral GA, Gomide NMP, Batista MPB, Píccolo PP, Teles TBG, Oliveira PM, Pereira AD. Sintomas depressivos em acadêmicos de Medicina da Universidade Federal de Goiás: um estudo de prevalência. Rev Psiquiatr Rio Gd Sul 2008; 30(2):124-130.

28. Alzahem AM, Van der Molen HT, Boer BJ. Effect of year of study on stress levels in male undergraduate dental students. Adv Med Educ Pract 2013; 4:217-222.

29. Miles S, Swift L, Leinster SJ. The Dundee Ready Education Environment Measure (DREEM): a review of its adoption and use. Med Teach 2012; 34(9):e620-e634.

30. Bakhshialiabad H, Bakhshi M, Hassanshahi G. Students' perceptions of the academic learning environment in seven medical sciences courses based on DREEM. Adv Med Educ Pract 2015; 6:195-203.

31. Guimaraes AGC, Falbo GH, Menezes T, Falbo A. Percepção do Estudante de medicina acerca do ambiente Educacional utilizando o DREEM. Rev Bras Edu Méd 2015; 39(4):517-526.

32. Tempski P, Santos IS, Mayer FB, Enns SC, Perotta BP, Paro HMS, Gannam S, Peleias M, Garcia VL, Baldas$\sin$ S, Guimaraes KB, Silva NR, Cruz EMTN, Tofoli LF, Silveira PSP, Martins MA. Relationship among Medical Student Resilience, Educational Environment and quality of life. PLoS One 2015; 10(6):e0131535.

33. Myint K, See-Ziau H, Husain R, Ismail R. Dental Students' Educational Environment and Perceived Stress: The University of Malaya Experience Malays. J Med Sc 2016; 23(3):49-56.

34. Poletto M, Koller SH. Contextos ecológicos: promotores de resiliência, fatores de risco e de proteção. Est Psicol 2008; 25(3):405-416.

35. Bacchi S, Licinio J. Resilience and Psychological Distress in Psychology and Medical Students. Acad Psychiatry. 2016; 41(2):185-188. 
36. Mendes KDS, Silveira RCCP, Galvão CM. Revisão integrativa: método de pesquisa para a incorporação de evidências na saúde e na enfermagem. Texto contexto - enferm 2008; 17(4):758-764.

37. Ganong LH. Integrative Reviews of Nursing Research. Res Nurs Health 1987; 10(1):1-11.

38. Pryjmachuk S, Richards DA. Predicting stress in pre-registration midwifery students attending a university in Northern England. Br J Midwifery 2008; 24(1):108-122.

39. Patel V, Araya R, Chowdhary N, King M, Kirkwood B, Nayak S, Simon G, Weiss HA. Detecting common mental disorders in primary care in India: a comparison of five screening questionnaires. Psychol Med 2008; 38(2):221-228.

40. Biro E, Balajti I, Adany R, Kosa K. Determinants of mental well-being in medical students. Soc Psychiat Epidemiol 2010; 45(2):253-258.

41. Bıro E, Balajti I, Adany R, Kosa K. Mental health and behaviour of students of public health and their correlation with social support: a cross-sectional study. BMC Public Health 2011; 11:871.

42. Pryjmachuk S, Richards DA. Predicting stress in pre-registration nursing students. Brit J Health Psychol 2007; 12(Pt 1):125-144.

43. Concepcion T, Barbosa C, Vélez JC, Pepper M, Andrade A, Gelaye B, Yanes D, Williams MA. Daytime Sleepiness, Poor Sleep Quality, Eveningness Chronotype and Common Mental Disorders Among Chilean College Students. J Am Coll Health 2014; 62(7):441-448.

44. Warbah L, Sathiyaseelan M, Vijayakumar C, Vasantharaj B, Russell S, Jacob KS. Psychological distress, personality, and adjustment among nursing students. Nurse Educ Today 2007; 27(6):597-601.

45. Rose D, Gelaye B, Sanchez S, Castañeda B, Sanchez E, Yanez ND, Williams MA. Morningness/eveningness chronotype, poor sleep quality, and daytime sleepiness in relation to common mental disorders among Peruvian college students. Psychol Health Med 2015; 20(3):345-352.

46. Liébana-Presa C, Fernández-Martí5nez E, Gándara AR, Muñoz-Villanueva MC, Vázquez-Casares AM, Rodríguez-Borrego MA. Psychological distress in health sciences college students and its relationship with academic engagement Rev Esc Enferm USP 2014; 48(4):715-722.

47. Gorter R, Freeman R, Hammen S, Murtomaa H, Blinkhorn A, Humphris G. Psychological stress and health in undergradate dental students: fifth year outcomes compared with first year baseline results from five European dental schools. Eur J Den Educ 2008; 12(2):6168.

48. Deasy C, Coughlan B, Pironom J, Jourdan D, Mannix-McNamara P. Psychological Distress and Coping amongst Higher Education Students: A Mixed Method Enquiry. PLoS One 2014; 9(12):e115193.

49. Harris RC, Millichamp CJ, Thomson WMNZ. Stress and coping in fourth-year medical and dental students. Dent J 2015; 111(3):102-108.
50. Costa EFO, Andrade TM, Silvany Neto AM, Melo EV Rosa AC, Alencar MA, Silva AM. Common mental disorders among medical students at Universidade Federal de Sergipe: a cross-sectional study. Rev Bras Psiquiatr 2010; 32(1):11-19.

51. Frey LL, Beesley D, Miller MR. Relational health, attachment, and psychological distress in college woman and men. Psychol Woman Q 2006; 30(3):303-311.

52. Pryjmachuk S, Richards DA. Mental health nursing students differ from other nursing students: Some observations from a study on stress and coping. Int J Ment Health Nurs Res 2007; 16(6):390-402.

53. Abdulghani HM. Stress and depression among medical students: a cross sectional study ate a medical college in Saudi Arabia. Pak J Med Sci 2008; 24(1):12-17.

54. Stallman HM. Prevalence of psychological distress in university students--implications for service delivery. Aust Fam Physician 2008; 37(8):673-677.

55. Tesfaye A. Prevalence and correlates of mental distress among regular undergraduate students of Hawassa University: a cross sectional survey. East Afr J Public Health 2009; 6(1):85-94.

56. Verger P, Combes J, Kovess-Masfly V, Choquet C, Guagliardo V, Rouillon F, Peretti-Wattel P. Psychological distress in first year university students: socioeconomic and academic stressors, mastery and social support in young men and women. Soc Psychiatr Epidemiol 2009; 44(8):643-665.

57. Park H, Heppner PP, Lee D. Maladaptive coping and self-esteem as mediators between perfectionism and pshycological distress. Pers Individ Dif 2010; 48(4):469474.

58. Abdulghani HM, AlKanhal AA, Mahmoud ES, Ponnamperuma GG, Alfaris EA. Stress and Its Effects on Medical Students: A Cross-sectional Study at a College of Medicine in Saudi Arabia. J Health Pop Nutr 2011; 29(5):516-522.

59. Gibbons C. Stress, coping and burn-out in nursing students. Int J Nurs Stud 2011; 47(10):1299-1309.

60. Byrd DR, McKinney KJ. Individual, Interpersonal, and Institutional Level Factors Associated With the Mental Health of College Students. J Am Coll Health 2012; 60(3):185-193.

61. Vázquez FL, Otero P, Díaz O. Psychological distress and related factors in female college students. J Am Coll Health 2012; 60(3):219-225.

62. Wei M, Liao KY, Heppner PP, Chao RC, Ku TY. Forbearance coping, identification with heritage culture, acculturative stress, and psychological distress among Chinese international students. J Couns Psychol 2012; 59(1):97-106.

63. Bastos JL, Barros AJD, Celeste RK, Paradies Y, Faerstein E. Age, class and race discrimination: their interactions and associations with mental health among Brazilian university students. Cad Saude Publica 2014, 30(1):175-186.

64. Carnicer JG, Calderón C. Empathy and Coping Strategies as Predictors of Well-Being in Spanish University Students Electronic. J Res Educ Psychol 2014; 12(1):129-146. 
65. Liu T, Wang Z, Zhou C, Li T. Affect and Self-Esteem as Mediators between Trait Resilience and Psychological Adjustment. Pers Individ Dif 2014; 66:92-97.

66. Saïas T, du Roscoät E, Véron L, Guignard R, Richard JB, Legleye S, Sauvade F, Kovess V, Beck F. Psychological distress in French college students: demographic, economic and social stressors. Results from the 2010 National Health Barometer. BMC Pub Health 2014; 14:256.

67. Dachew BA, Bisetegn TA, Gebremariam RB. Prevalence of Mental Distress and Associated Factors among Undergraduate Students of University of Gondar, Northwest Ethiopia: A Cross-Sectional Institutional Based Study. PLoS One 2015; 10(3):e0119464.

68. Budescu M, Silverman LR. Kinship Support and Academic Efficacy Among College Students: A Cross-Sectional Examination. J Child Fam Stud 2016; 25(6):17891801.

69. Deasy C, Coughlan B, Pironom J, Jourdan D, Mannix-McNamara P. Predictors of health of pre-registration nursing and midwifery students: Findings from a cross-sectional survey. Nurse Educ Today 2016; 36:427433.

70. Wang Z, Koenig HG, Ma H, Shohaib SA. Religion, Purpose in Life, Social Support, and Psychological Distress in Chinese University Students. J Relig Health 2016; 55:1055.

71. Dyrbye LN, Thomas MR, Shanafelt TD. Systematic Review of Depression, Anxiety, and Other Indicators of Psychological Distress Among U.S. and Canadian Medical Students. Acad Med 2006; 81(4):354-373.

72. Carlotto MS, Amazarray MR, Chinazzo I, Taborda L. Transtornos mentais comuns e fatores associados em trabalhadores: uma análise na perspectiva de gênero. Cad Saúde Colet 2011; 19(2):172-178.

73. El Ansari W, Oskrochi R, Haghgoo G. Are students' symptoms and health complaints associated with perceived stress at university? Perspectives from the United Kingdom and Egypt. Int J Environ Res Public Health 2014; 11(10):9981-10002.

74. Elani HW, Allisson PJ, Kumar RA, Mancini L, Lambrou A, Bedos C. A sistematic review of stress in dental students. J Dent Educ 2014; 78(2):226-242.

75. Rotenstein LS, Ramos MA, Torre M, Sega JB, Peluso MJ, Guille C, Sen S, Mata DA. Prevalence of Depression, Depressive Symptoms, and Suicidal Ideation Among Medical Students: A Systematic Review and Meta-Analysis. JAMA 2016; 316(21):2214-2236.
76. Dyrbye LN, Massie FSJr, Eacker A, Harper W, Power D, Durning SJ, Thomas MR, Moutier C, Satele D, Sloan J, Shanafelt TD. Relationship between burnout and professional conduct and attitudes among US medical students. JAMA 2010; 304(11):1173-1180.

77. Folkman S, Lazarus RS, Gruen RJ, DeLongis A. Appraisal, coping, health status, and psychological symptoms. J Pers Soc Psychol 1986; 50(3):571-579.

78. Lazarus RS, Folkman S. Stress, appraisal and coping. New York: Springer; 1984.

79. Alburn G, Gott M, Hoare K. What is resilience? An Integrative Review of the empirical literature. J Adv Nurs 2016; 72(5):980-1000.

80. Campbell-Sills L, Cohan SL, Stein MB. Relationship of resilience to personality, coping, and psychiatric symptoms in young adults. Beh Res Ther 2005; 44(4):585599.

81. Lima MCP. Sobre trote, vampiros e relacionamento humano nas escolas médicas. Rev Bras Educ Med 2012; 36(3):407-413.

82. Costa SM, Dias OV, Dias ACA, Souza TR, Canela JR. Trote universitário: diversão ou constrangimento entre acadêmicos da saúde? Rev Bioét 2013; 21(2):350-358.

83. Paradies Y. A systematic Review of empirical research on self-reported racism and health. Int J Epidemiol 2006; 35:888-901.

84. Pascoe EA, Smart-Richman L. Perceived discrimination and health: a meta-analytic review. Psychol Bull 2009; 135(4):531-554.

Artigo apresentado em 05/05/2017

Aprovado em 26/07/2017

Versão final apresentada em 28/07/2017 